\title{
TRIFLUOROACETIC ACID CATALYZED ONE-POT FOUR-COMPONENT DOMINO REACTION FOR THE SYNTHESIS OF SUBSTITUTED DIHYDRO 2-OXYPYRROLES
}

\author{
MOJTABA LASHKARI ${ }^{a}$, MALEK TAHER MAGHSOODLOU ${ }^{b, *}$, \\ MAHSA KARIMA ${ }^{b}$, MEHRNOOSH KANGANI $^{b}$ \\ ${ }^{a}$ Faculty of Science, Velayat University, Iranshahr, Iran \\ ${ }^{b}$ Department of Chemistry, University of Sistan and Baluchestan, P.O. Box 98135 - 674, Zahedan, Iran
}

\section{ABSTRACT}

Trifluoroacetic acid was applied as an efficient catalyst for the one-pot four-component synthesis of N-aryl/alkyl-3-aminodihydropyrrol-2-one-4-carboxylates via the domino reaction of amines, formaldehyde and dialkyl acetylenedicarboxylates at ambient temperature in methanol. This methodology includes number of advantages such as: short reaction time, clean work-up, use of inexpensive catalyst, high yields and clean work-up. The work-up of this reaction involves only a filtration and a simple washing step with $\mathrm{MeOH}$, and there is no need for column chromatography.

Keywords: Trifluoroacetic acid; N-aryl/alkyl-3-aminodihydropyrrol-2-one-4-carboxylates; ambient temperature

\section{INTRODUCTION}

In recent years, growing attention has been paid to the synthesis of N-heterocycles due to diverse biological and pharmaceutical applications [1$3]$. In this respect, the presence of pyrrol-2-ones (5-lactams or $\gamma$-lactams) in pharmaceuticals and natural products has continued to stimulate a great deal of interest in the development of new methodologies for their synthesis [4-6]. There are several bioactive natural molecules with a pyrrol-2-one moiety, such as holomycin and thiolutin [7], thiomarinol A4 [8], oteromycin [9], pyrrocidine A [10], quinolactacin C [11], and ypaoamide [12]. On the other hand, dihydropyrrol-2-ones have been successfully used as peptidomimetic [13], HIV integrase [14], herbicidals [15], DNA polymerase inhibitors [16], caspase-3 inhibitors [17] cytotoxic and antitumor agents [18], antibiotics [19], and also inhibitors of the annexin A2-S100A10 protein interaction [20]. Recently, a few methods have been reported for the synthesis of highly substituted dihydropyrrol-2-ones using one-pot, four-component reactions in the presence of catalyst, such as $\mathrm{AcOH}$ [21], $\mathrm{I}_{2}$ [22], benzoic acid [23], $\mathrm{TiO}_{2}$ nanopowder [24] or $\mathrm{Cu}(\mathrm{OAc})_{2} \cdot \mathrm{H}_{2} \mathrm{O}$ [25]. However, some of these methods have drawbacks, such as high temperature and utilize a chlorinated solvent. Therefore, the development of a milder and more efficient route for one-pot synthesis of these important heterocycles is still in demand. In continue of our ongoing program on multi-component reactions [26-31], an efficient and convenient synthesis of $\mathrm{N}$-aryl-3-aminodihydropyrrol-2-one-4-carboxylates has been accomplished using trifluoroacetic acid as an efficient catalyst in $\mathrm{MeOH}$ at ambient temperature, with good yields (Schemes 1). Trifluoroacetic acid is widely utilized in organic synthesis as a solvent [32] or as an acid catalyst for different organic transformations such as rearrangements [33], functional group deprotections [34], reductions [35], oxidations [36], hydroarylations [37], condensations [38], and also trifluoromethylation reactions [39].<smiles>[R]OCC#CC(=O)O[R2]</smiles>

1
2<smiles>[17N][18NH]C=O</smiles>
3

\section{$\mathrm{CF}_{3} \mathrm{CO}_{2} \mathrm{H}(20 \mathrm{~mol} \mathrm{\%})$} $\mathrm{MeOH}$, r.t.<smiles>[R20]C([R20])=C1C(=O)CN([Al])C1=O</smiles>

5

Scheme 1. Synthesis of N-aryl-3-aminodihydropyrrol-2-one-4-carboxylates in the presence of trifluoroacetic acid as catalyst in MeOH at ambient temperature

\section{RESULTS AND DISCUSSIN}

Formaldehyde, aniline, and dimethyl acetylenedicarboxylate were taken as model compounds for the optimization of the reaction conditions. For this purpose, the reaction was initially carried out in different conditions (Table 1). As can be seen, trifluoroacetic acid (10 mol \%) was found to be the most effective catalyst for the reaction at room temperature. 
Table 1. Optimization of the reaction conditions for the synthesis of $5 \mathrm{a}^{a}$

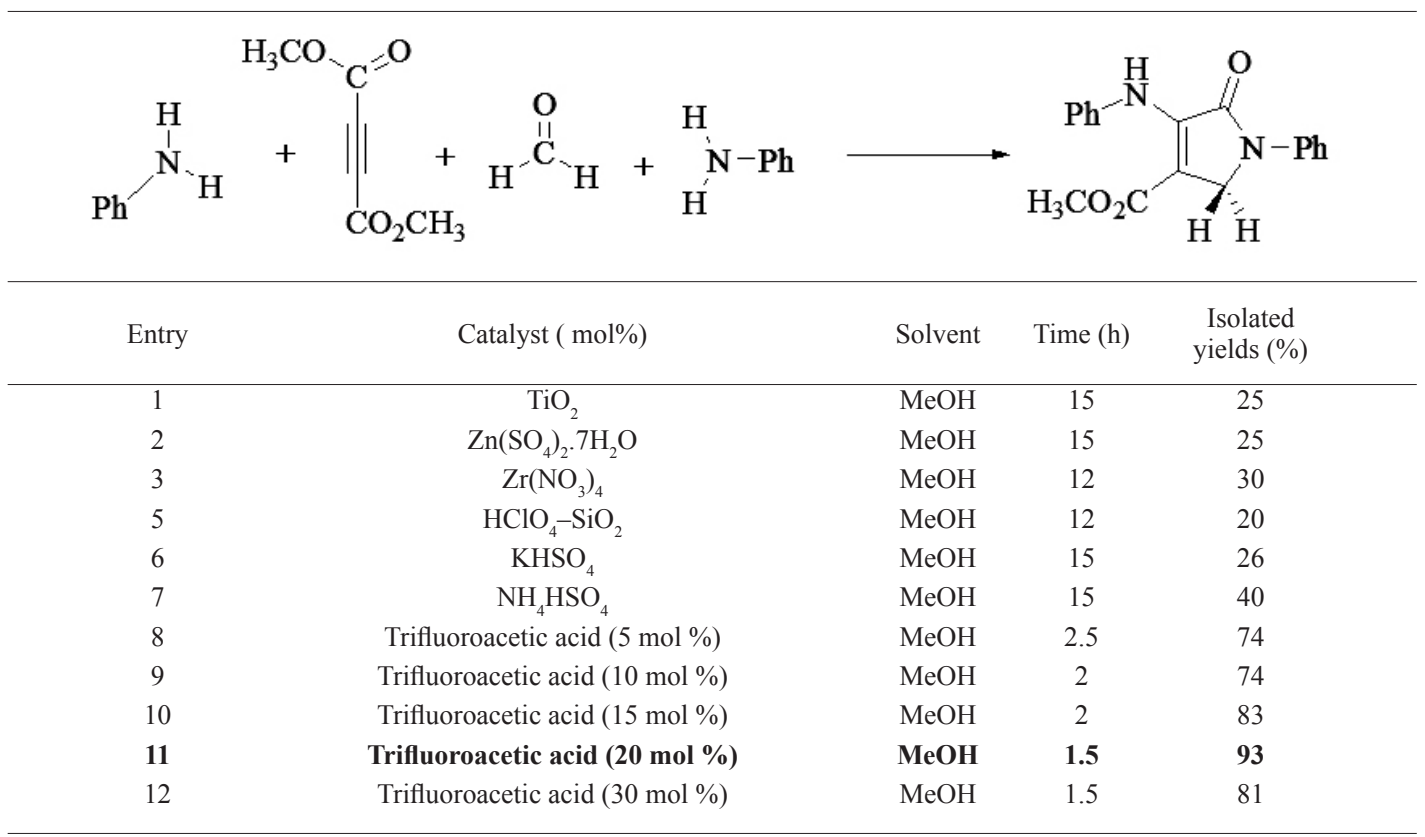

${ }^{a}$ Reaction conditions: aniline $(2.0 \mathrm{mmol})$, DMAD $(1.0 \mathrm{mmol})$, and formaldehyde $(1.5 \mathrm{mmol})$ at room temperature in the presence of catalyst

To demonstrate the utility and generality of this method, the various substituted anilines, dimethyl and/or diethyl acetylenedicarboxylate and formaldehyde were employed successfully to generate the desired N-aryl-3aminodihydropyrrol-2-one-4-carboxylates 5a-h (Table 2). Encouraged by these results, different poly functionalized dihydropyrrol-2-ones $\mathbf{5 i - q}$ were synthesized using two different amines. Aliphatic amines, such as benzyl amine, 1-(pyridin-2-yl)methanamine and $n$-butyl amine, were reacted with dialkyl acetylenedicarboxylates, anilines and formaldehyde to produce the corresponding products in good to high yields.

Table 2. Synthesis of N-aryl-3-aminodihydropyrrol-2-one-4-carboxylates 5.

\begin{tabular}{|c|c|c|c|c|c|c|c|c|}
\hline Entry & Product & $\mathrm{R}^{1}$ & $\mathrm{R}^{2}$ & $\mathrm{Ar}$ & Time (h) & Yield $(\%)^{a}$ & $\mathrm{~m} \cdot \mathrm{p}\left({ }^{\circ} \mathrm{C}\right)$ & Lit.mp $\left({ }^{\circ} \mathrm{C}\right)[\operatorname{Ref}]$ \\
\hline 1 & $5 \mathbf{a}$ & $\mathrm{Ph}$ & $\mathrm{Me}$ & $\mathrm{Ph}$ & 5 & 91 & $156-157$ & $155-156[22]$ \\
\hline 2 & $5 \mathbf{b}$ & $4-\mathrm{Me}-\mathrm{C}_{6} \mathrm{H}_{4}$ & $\mathrm{Me}$ & 4-Me- $\mathrm{C}_{6} \mathrm{H}_{4}$ & 6 & 85 & $177-179$ & $177-178$ [22] \\
\hline 34 & $5 c$ & $\mathrm{Ph}$ & Et & $\mathrm{Ph}$ & 5 & 89 & $138-140$ & $138-140[21]$ \\
\hline 5 & $5 d$ & 4-Me- $\mathrm{C}_{6} \mathrm{H}_{4}$ & Et & $4-\mathrm{Me}-\mathrm{C}_{6} \mathrm{H}_{4}$ & 7 & 85 & $128-130$ & $131-132$ [21] \\
\hline 6 & $5 e$ & $4-\mathrm{OMe}-\mathrm{C}_{6} \mathrm{H}_{4}$ & $\mathrm{Et}$ & $4-\mathrm{OMe}-\mathrm{C}_{6} \mathrm{H}_{4}$ & 7 & 92 & $152-154$ & $152-154[31]$ \\
\hline 7 & $5 f$ & $4-\mathrm{Br}-\mathrm{C}_{6} \mathrm{H}_{4}$ & Et & $4-\mathrm{Br}-\mathrm{C}_{6} \mathrm{H}_{4}$ & 7 & 91 & $171-172$ & $169-171[21]$ \\
\hline 9 & $5 h$ & $4-\mathrm{Cl}-\mathrm{C}_{6} \mathrm{H}_{4}$ & $\mathrm{Et}$ & $4-\mathrm{Cl}-\mathrm{C}_{6} \mathrm{H}_{4}$ & 6 & 85 & $167-170$ & $168-170[30]$ \\
\hline 10 & $5 \mathbf{i}$ & $\mathrm{PhCH}_{2}$ & $\mathrm{Me}$ & $\mathrm{Ph}$ & 7 & 84 & $140-141$ & $140-141[21]$ \\
\hline 11 & $5 \mathbf{j}$ & $\mathrm{PhCH}_{2}$ & $\mathrm{Me}$ & $4-\mathrm{F}-\mathrm{C}_{6} \mathrm{H}_{4}$ & 7 & 85 & $168-170$ & $166-168[31]$ \\
\hline 12 & $5 k$ & $\mathrm{PhCH}_{2}^{2}$ & Et & $\mathrm{Ph}$ & 8 & 77 & $127-129$ & $129130[21]$ \\
\hline 13 & 51 & $\mathrm{PhCH}_{2}^{2}$ & $\mathrm{Me}$ & $4-\mathrm{Br}^{-} \mathrm{C}_{6} \mathrm{H}_{4}$ & 12 & 76 & $119-121$ & $120-121[22]$ \\
\hline 14 & $5 \mathrm{~m}$ & $\mathrm{PhCH}_{2}$ & $\mathrm{Me}$ & $4-\mathrm{Me}-\mathrm{C}_{6} \mathrm{H}_{4}$ & 6 & 85 & $144-146$ & $144-146[30]$ \\
\hline 15 & $5 n$ & $\mathrm{C}_{5} \mathrm{H}_{4} \mathrm{~N}-{ }_{2}-\mathrm{CH}_{2}$ & $\mathrm{Me}$ & $4-\mathrm{Me}-\mathrm{C}_{6} \mathrm{H}_{4}^{4}$ & 6 & 80 & $104-106$ & $106-108$ [29] \\
\hline 17 & $5 p$ & $\mathrm{n}-\mathrm{C}_{4} \mathrm{H}_{9}$ & Et & $4-\mathrm{Br}-\mathrm{C}_{6} \mathrm{H}_{4}$ & 6 & 85 & $94-97$ & $94-96[30]$ \\
\hline 18 & $5 q$ & $4-\mathrm{F}-\mathrm{C}_{6} \mathrm{H}_{4}$ & Et & $4-\mathrm{F}-\mathrm{C}_{6} \mathrm{H}_{4}^{4}$ & 6 & 89 & $170-172$ & $172-173[21]$ \\
\hline 19 & $5 r$ & $4-\mathrm{Cl}-\mathrm{C}_{6} \mathrm{H}_{4}^{4}$ & Et & $\mathrm{Me}-\mathrm{C}_{6} \mathrm{H}_{4}{ }_{4}^{4}$ & 5 & 93 & $169-170$ & $168-170[30]$ \\
\hline 20 & $5 s$ & $4-\mathrm{Cl}-\mathrm{C}_{6} \mathrm{H}_{4}$ & $\mathrm{Me}$ & $4-\mathrm{Cl}-\mathrm{C}_{6} \mathrm{H}_{4}$ & 6 & 90 & $174-175$ & $173-174[22]$ \\
\hline 21 & $5 t$ & $\mathrm{PhCH}_{2}^{4}$ & $\mathrm{Me}$ & $3,4-\mathrm{Cl}_{2}-\mathrm{C}_{6} \mathrm{H}_{3}$ & 8 & 89 & $165-167$ & $162-164[40]$ \\
\hline 22 & $5 \mathbf{u}$ & $\mathrm{PhCH}_{2}$ & $\mathrm{Me}$ & $4-\mathrm{Cl}-\mathrm{C}_{6} \mathrm{H}_{4}$ & 6 & 93 & $143-146$ & $147-147[22]$ \\
\hline 23 & $5 v$ & $\mathrm{PhCH}_{2}^{2}$ & $\mathrm{Me}$ & $4-\mathrm{OMe}-\mathrm{C}_{6} \mathrm{H}_{4}$ & 8 & 84 & $123-126$ & $129-130[22]$ \\
\hline 24 & $5 w$ & $4-\mathrm{Br}-\mathrm{C}_{6} \mathrm{H}_{4}$ & $\mathrm{Me}$ & $4-\mathrm{Br}-\mathrm{C}_{6} \mathrm{H}_{4}$ & 6 & 90 & $176-179$ & $179-180[22]$ \\
\hline 25 & $5 x$ & $4-\mathrm{Br}-\mathrm{C}_{6} \mathrm{H}_{4}$ & $\mathrm{Me}$ & $4-\mathrm{Cl}-\mathrm{C}_{6} \mathrm{H}_{4}$ & 6 & 89 & $157-160$ & $160-162[41]$ \\
\hline 26 & $5 y$ & $\mathrm{Ph}$ & Et & $4-\mathrm{Cl}-\mathrm{C}_{6} \mathrm{H}_{4}^{4}$ & 7 & 84 & 199 & 202 [42] \\
\hline
\end{tabular}

${ }^{a}$ Isolated Yield

base on the literature, this synthetic method can be combination of Michael, formation of imine, Mannich-type and cyclization reactions (Scheme 3). 
<smiles>[R]OC(=O)C#CC(=O)O[R2]</smiles><smiles>O=C[14CH2]N[Al]</smiles>

$\mathbf{C}$

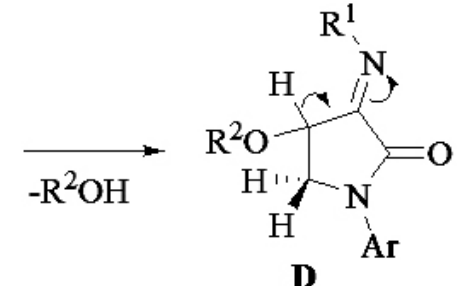

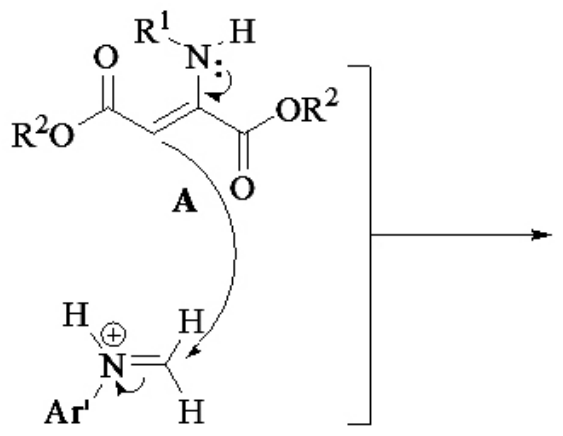

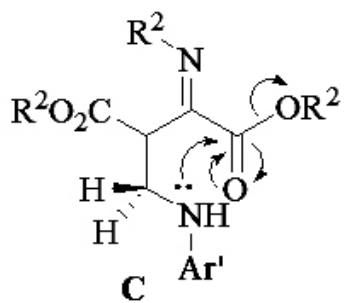

\section{tautomerization}<smiles>[R4]NC1=C([R20])CN([Y19])C1=O</smiles>

5

Scheme 3. Proposed mechanism for the synthesis of N-aryl-3-aminodihydropyrrol-2-one-4-carboxylates 5

To compare the efficiency and applicability of $\mathrm{CF}_{3} \mathrm{CO}_{2} \mathrm{H}$ with the reported catalysts and conditions in the literature for the synthesis of $\mathrm{N}$-aryl-3aminodihydropyrrol-2-one-4-carboxylates, we have tabulated the results of these catalysts in Table 3 . As shown in Table $3, \mathrm{CF}_{3} \mathrm{CO}_{2} \mathrm{H}_{\mathrm{can}}$ act as efficient catalyst with respect to reaction times and yields of products.

Table 3. Comparison result of $\mathrm{CF}_{3} \mathrm{CO}_{2} \mathrm{H}$ with the reported catalysts in literature for the synthesis of N-aryl-3-aminodihydropyrrol-2-one-4-carboxylates 5c.

\begin{tabular}{|c|c|c|c|c|c|}
\hline Entry & Catalyst & Conditions & Time (h) & Yield (\%) & Ref. \\
\hline 1 & $\mathrm{Cu}(\mathrm{OAc})_{2} \cdot \mathrm{H}_{2} \mathrm{O}$ & $\mathrm{MeOH}, \mathrm{rt}$ & 5 & 85 & 25 \\
\hline 2 & $\mathrm{I}_{2}$ & $\mathrm{MeOH}, \mathrm{rt}$ & 1 & 81 & 22 \\
\hline 3 & $\mathrm{AcOH}$ & $\mathrm{EtOH} / 70{ }^{\circ} \mathrm{C}$ & 4 & 85 & 21 \\
\hline 4 & Oxalic acid dehydrate & $\mathrm{MeOH}, \mathrm{rt}$ & 2 & 87 & 43 \\
\hline 5 & {$[$ Hpyro $]\left[\mathrm{HSO}_{4}\right]$} & $\mathrm{MeOH}, \mathrm{rt}$ & 6 & 80 & 31 \\
\hline 6 & $\mathrm{Al}\left(\mathrm{H}_{2} \mathrm{PO}_{4}\right)_{3}$ & $\mathrm{MeOH}, \mathrm{rt}$ & 5 & 80 & 29 \\
\hline 7 & $\mathrm{InCl}_{3}$ & $\mathrm{MeOH}, \mathrm{rt}$ & 3 & 85 & 44 \\
\hline 8 & {$\left[\mathrm{n}-\mathrm{Bu}_{4} \mathrm{~N}\right]\left[\mathrm{HSO}_{4}\right]$} & $\mathrm{MeOH}, \mathrm{rt}$ & 4 & 86 & 30 \\
\hline 9 & $\mathrm{CF}_{3} \mathrm{CO}_{2} \mathrm{H}$ & $\mathrm{MeOH}, \mathrm{rt}$ & 5 & 89 & This work \\
\hline
\end{tabular}

\section{EXPERIMENTAL}

Melting points were taken on an Electrothermal 9100 apparatus. IR spectra were obtained on a JASCO FT/IR-460 plus spectrometer. The ${ }^{1} \mathrm{H}$ NMR and ${ }^{13} \mathrm{C}$ NMR spectra were recorded on a Bruker DRX-400 Avanve instrument with $\mathrm{CDCl}_{3}$ as solvent and using TMS as internal reference at $400 \mathrm{MHz}$ and $100 \mathrm{MHz}$, respectively. Chemicals were purchased from Merck (Darmastadt, Germany), Acros (Geel, Belgium) and Fluka (Buchs, Switzerland), and used without further purification.

General procedure for the synthesis of $\mathrm{N}$-aryl-3-aminodihydropyrrol-2one-4-carboxylates 5

A mixture of amine $1(1.0 \mathrm{mmol})$ and dialkyl acetylenedicarboxylate 2 (1.0 mmol) in MeOH (3 mL) was stirred for $25 \mathrm{~min}$. Next, amine 3 (1.0 mmol), formaldehyde 4 (37\% solution, $1.5 \mathrm{mmol})$ and trifluoroacetic acid (20 mol \%) were added in successively. The reaction mixture was allowed to stir at ambient temperature for appropriate time. After completion of the reaction (monitored by TLC), the water was added to produce solid precipitate, and the precipitate was filtered off and washed with methanol $(3 \times 2 \mathrm{~mL})$ to give the pure product 5. The structures of the synthesized compounds were characterized by their IR, ${ }^{1} \mathrm{H}$ NMR and ${ }^{13} \mathrm{C}$ NMR spectra and were found to be identical with data described in the literature [21,22].

Characterization data of some compounds

Methyl 2,5-dihydro-5-oxo-1-phenyl-4-(phenylamino)-1H-pyrrole-3carboxylate (5a)

White solid, IR $(\mathrm{KBr})\left(\delta \max , \mathrm{cm}^{-1}\right): 3310(\mathrm{NH}), 1705,1684,1645 ;{ }^{1} \mathrm{H}$ NMR (400 MHz, $\left.\mathrm{CDCl}_{3}\right): \delta 3.76\left(3 \mathrm{H}, \mathrm{s}, \mathrm{OCH}_{3}\right), 4.57\left(2 \mathrm{H}, \mathrm{s}, \mathrm{CH}_{2}\right), 7.16-7.23$ $(4 \mathrm{H}, \mathrm{m}, \mathrm{ArH}), 7.34(2 \mathrm{H}, \mathrm{t}, J=8.0 \mathrm{~Hz}, \mathrm{ArH}), 7.42(2 \mathrm{H}, \mathrm{t}, J=8.0 \mathrm{~Hz}, \mathrm{ArH})$, $7.81(2 \mathrm{H}, \mathrm{d}, J=8.0 \mathrm{~Hz}, \mathrm{ArH}), 8.05(1 \mathrm{H}$, br s, NH).

Ethyl 4-(p-tolylamino)-2,5-dihydro-5-oxo-1-p-tolyl-1H-pyrrole-3carboxylate ( $5 \boldsymbol{d})$

Yellow solid, IR $(\mathrm{KBr})\left(\delta \max , \mathrm{cm}^{-1}\right): 3310(\mathrm{NH}), 1707,1682,1649 ;{ }^{1} \mathrm{H}$ NMR (400 MHz, CDCl $): \delta 1.25\left(3 \mathrm{H}, \mathrm{t}, J=7.2 \mathrm{~Hz}, \mathrm{OCH}_{2} \mathrm{CH} 3\right), 2.36(3 \mathrm{H}, \mathrm{s}$, $\left.\mathrm{CH}_{3}\right), 2.37\left(3 \mathrm{H}, \mathrm{s}, \mathrm{CH}_{3}\right), 4.23\left(2 \mathrm{H}, \mathrm{t}, J=7.2 \mathrm{~Hz}, \mathrm{OCH}_{2} \mathrm{CH}_{3}\right), 4.52\left(2 \mathrm{H}, \mathrm{s}, \mathrm{CH}_{2}\right)$, $7.06(2 \mathrm{H}, \mathrm{d}, J=8.4 \mathrm{~Hz}, \mathrm{ArH}), 7.14(2 \mathrm{H}, \mathrm{d}, J=8.0 \mathrm{~Hz}, \mathrm{ArH}), 7.21(2 \mathrm{H}, \mathrm{d}, J=$ $8.4 \mathrm{~Hz}, \mathrm{ArH}), 7.69(2 \mathrm{H}, \mathrm{d}, J=8.8 \mathrm{~Hz}, \mathrm{ArH}), 8.01\left(1 \mathrm{H}\right.$, brs, NH) $;{ }^{13} \mathrm{C} \mathrm{NMR}(100$ $\left.\mathrm{MHz}, \mathrm{CDCl}_{3}\right): \delta 14.2,20.9,21.0,48.3,60.2,102.4,119.1,122.9,128.9,129.6$, 134.2, 134.6, 136.2, 136.3, 143.1, 163.7, 164.7.

Methyl 4-(benzylamino)-1-p-tolyl-2,5-dihydro-5-oxo-1H-pyrrole-3carboxylate $(5 \mathrm{~m})$.

White solid IR $(\mathrm{KBr})\left(\delta \max , \mathrm{cm}^{-1}\right): 3310(\mathrm{NH}), 1704,1682,1646 ;{ }^{1} \mathrm{H}$ NMR (400 MHz, CDCl3): $\delta 1.34$ (3H, t, $\left.J=7.2 \mathrm{~Hz}, \mathrm{OCH}_{2} \mathrm{CH}_{3}\right), 4.27(2 \mathrm{H}, \mathrm{t}, J$ 
$\left.=7.2 \mathrm{~Hz}, \mathrm{OCH}_{2} \mathrm{CH}_{3}\right), 4.41\left(2 \mathrm{H}, \mathrm{s}, \mathrm{CH}_{2}-\mathrm{N}\right), 5.12\left(2 \mathrm{H}, \mathrm{d}, J=6.4 \mathrm{~Hz}, \mathrm{CH}_{2}-\mathrm{NH}\right)$, $6.90(1 \mathrm{H}, \mathrm{br}, \mathrm{NH}), 7.28-7.37(5 \mathrm{H}, \mathrm{m}, \mathrm{ArH}), 7.52(2 \mathrm{H}, \mathrm{d}, J=8.8 \mathrm{~Hz}, \mathrm{ArH}), 7.70$ $(2 \mathrm{H}, \mathrm{d}, J=8.8 \mathrm{~Hz}, \mathrm{ArH}) ;{ }^{13} \mathrm{C} \mathrm{NMR}\left(100 \mathrm{MHz}, \mathrm{CDCl}_{3}\right): \delta 20.9,46.6,48.0,51.0$, 97.1, 119.4, 127.3, 127.5, 128.7, 129.6, 134.8, 136.2,139.5, 164.3, 165.6.

\section{CONCLUSION}

In conclusion we have identified an efficient and simple one-pot reaction for the synthesis of N-aryl-3-aminodihydropyrrol-2-one-4-carboxylates derivatives using trifluroacetic acid as an economic catalyst at ambient conditions. This methodology has several advantages such as simplified workup procedures, mild conditions, and high yields

\section{ACKNOWLEDGMENT}

We gratefully acknowledge financial support from the Research Council of the University of Sistan and Baluchestan.

\section{REFERENCES}

1. T. Eicher, S. Hauptmann, Wiley-VCH, Weinheim, (2003).

2. P.Z. Zhang, S.F. Zhou, T.R. Li, L. Jiang, Chin. Chem. Lett. 23, 1381 (2012)

3. T.N. Akhaja, J.P. Raval, Design, Chin. Chem. Lett. 23, 446 (2012)

4. W.J. Bai, S.K. Jakson, T.R.R. Pettus, Org. Lett. 14, 3862 (2012)

5. M. Aginagalde, T. Bello, C. Masdeu, Y.Vara, A. Arrieta, F. Cossío J. Org. Chem. 75, 7435 (2010)

6. H. Anaraki-Ardakani, M. Noei, A. Tabarzad, Chin. Chem. Lett. 23, 45 (2012)

7. L. Ettlinger, E. Ga"uemann, R. Hu"tter, W. Keller-Schierlein, F. Kradolfer, L. Neipp, V. Prelog, H. Zähner, Helv. Chim. Acta 42, 563 (1959)

8. H. Shiozawa, S. Takahashi, J. Antibiot. 47, 851 (1994)

9. S.B. Singh, M.A. Goetz, E.T. Jones, G. F. Bills, R. A. Giacobbe, L.H Siobhan Stevens- Miles, D. L. Williams, J. Org. Chem. 60, 7040 (1995)

10. H. He, H.Y. Yang, R. Bigelis, E. H. Solum, M. Greenstein, Tetrahedron Lett. 43, 1633 (2002)

11. A.J. Clark, C.P. Dell, J.M. McDonagh, J. Geden, P. Mawdsley, Org. Lett. 5, 2063 (2003)

12. J. Chen, P.Q. Huang, Y. Queneau, J. Org. Chem. 74, 7457 (2009)

13. A. Raghuraman, E. Ko, L.M. Perez, T. R. Ioerger, K. Burgess, J. Am. Chem. Soc. 133, 12350 (2011)

14. T. Kawasuji, M. Fuji, T. Yoshinaga, A. Sato, T. Fujiwara, R. Kiyama, Bioorg. Med. Chem. 15, 5487 (2007)

15. L. Zhang, Y. Tan, N.X. Wang, Q.Y. Wu, Z. Xi, G.F. Yang, Bioorg. Med. Chem. 18, 7948 (2010)

16. Y. Mizushina, S. Kobayashi, K. Kuramochi, S.Nagata, F. Sugawara, K. Sakaguchi, Biochem.Biophys. Res. Commun. 273, 784 (2000)

17. Q. Zhu, L. Gao, Z. Chen, S. Zheng, H. Shu, J. Liet, Eur. J. Med. Chem 54, $232(2012)$

18. B. Li, M.P.A. Lyle, G. Chen, J. Li, K. L. T. Hu, M. A. Alaoui-Jamali, J. Webster, Bioorg. Med. Chem. 15, 4601 (2007)

19. A.S. Demir, F. Aydigan, I.M. Akhmedov, Tetrahedron: Asymmetry 13, $601(2002)$

20. T.R.K. Reddy, C. L, X. Guo, X. Myrvang, H.K. P. M. Fischer, L. V. Dekker, J. Med. Chem. 54, 2080 (2011)

21. Q. Zhu, H. Jiang, J. Li, S. Liu, C. Xia, M. Zhang, J. Comb. Chem. 11, 685 (2009)

22. A.T. Khan, A. Ghosh, Md.M. Khan, Tetrahedron Lett. 53, 2622 (2012)

23. H. Gao, J. Sun, C.G. Yan, Tetrahedron 69, 589 (2013)

24. S. Rana, M. Brown, A. Dutta, A. Bhaumik, C. Mukhopadhyay, Tetrahedron Lett. 54, 1371 (2013)

25. L. Lv, S. Zheng, X. Cai, Z. Chen, Q. Zhu, S. Liu, ACS Comb. Sci. 15, $183(2013)$

26. R. Doostmohammadi, M.T. Maghsoodlou, N. Hazeri, S.M. HabibiKhorassani, Res. Chem. Intermed., 38, 4061 (2012)

27. S.S. Sajadikhah, M.T. Maghsoodlou, N. Hazeri, S. M. Habibi-Khorassani, A.C. Willis, Chin. Chem. Lett. 23, 569 (2012)

28. M.T. Maghsoodlou, S.M. Habibi-Khorasani, Z. Shahkarami, N. Maleki, M. Rostamizadeh, Chem. Lett. 21, 686 (2010)

29. S.S. Sajadikhah, N. Hazeri, M.T. Maghsoodlou, S.M. Habibi-Khorassani, A. Beigbabaei, A.C. Willis, J. Iran. Chem. Soc. 10, 863 (2013)

30. S.S. Sajadikhah, N. Hazeri, Res. Chem. Int. 40, 737(2014)

31. S.S. Sajadikhah, N. Hazeri, M.T. Maghsoodlou, S.M. Habibi-Khorassani,
J. Chin. Chem. Soc. 60, 1003 (2013)

32. For a review, see: (a) T. Wu, H. Yu, C. Li, ARKIVOC ix (2004) 60 65; (b) P.E. Peterson, C. Casey, E.V.P. Tao, A. Agtarap, G. Thompson, J. Am. Chem. Soc. 87 (1965) 5163-5169; (c) P.E. Peterson, R.J. Bopp, D.M. Chevli, E.L. Curran, D.E. Dillard, R.J. Kamat, J. Am. Chem. Soc. 89 (1967) 5902-5911

33. (a) For a review, see: K. Turnbull, D.M. Krein, Synthesis (1999) 391-392; (b) R. Venkateswarlu, C. Kamakshi, P.V. Subhash, S.G.A. Moinuddin, M.P. Gowri, R.S. Ward, A. Pelter, M.B. Hursthouse, S.J. Coles, M.E Light, Tetrahedron 61 (2005) 8956-8961; (c) A.M. Martin-Castro, Chem. Rev. 104 (2004) 2939-3002; (d) R. Pathak, S. Madapa, S. Batra, Tetrahedron 63 (2007) 451-460.

34. For a review, see: (a) R.D. Wakharkar, M.B. Sahasrabuddhe, H.B Borate, M.K. Gurjar, Synthesis (2004) 1830-1834; (b) V.V.N.K.V.P. Raju, V. Ravindra, S.S. Kamath, V.T. Mathad, P.K. Dubey, P.P. Reddya, ARKIVOC xii (2009) 296-301; (c) A.K. Singh, R.E. Weaver, G.L. Powers, V.W. Rosso, C. Wei, D.A. Lust, A.S. Kotnis, F.T. Comezoglu, M. Liu, K.S. Bembenek, B.D. Phan, D.J. Vanyo, M.L. Davies, R. Mathew, V.A. Palaniswamy, W.-S. Li, K. Gadamsetti, C.J. Spagnuolo, W.J. Winter, Org. Proc. Res. Dev. 7 (2003) 25-27

35. For a review, see: (a) S.A. Bowden, J.N. Burke, F. Gray, S. McKown, J.D. Moseley, W.O. Moss, P.M. Murray, M.J. Welham, M.J. Young, Org. Proc. Res. Dev. 8 (2004) 33-44; (b) G.T. Bourne, D.J. Kuster, G.R. Marshall, Chem. Eur. J. 16 (2010) 8439-8445; (c) R.N. Misra, D.B. Rawlins, H. Y. Xiao, W. Shan, I. Bursuker, K.A. Kellar, J.G. Mulheron, J.S. Sack, J.S. Tokarski, S.D. Kimball, K.R. Webster, Bioorg. Med. Chem. Lett. 13 (2003) 1133-1136; (d) K.G. Akamanchi, N.R. Varalakshmy, B.A. Chaudhari, Synlett (1997) 371-372; (e) M.N. Masuno, T.F. Molinski, Tetrahedron Lett. 42 (2001) 8263-8266; (f) J.P. Patel, A.-H. Li, H. Dong, V.L. Korlipara, M.J. Mulvihill, Tetrahedron Lett. 50 (2009) 5975-5977.

36. For a review, see: (a) A.A. Zagulyaeva, M.S. Yusubov, V.V. Zhdankin, J. Org. Chem. 75 (2010) 2119-2122; (b) G.A. Olah, Q. Wang, N.J. Trivedi, G.K.S. Prakash, Synthesis (1991) 739-740; (c) R.A. Kjonaas, A.E. Clemons, J. Chem. Ed. 85 (2008) 827-828

37. For a review, see: (a) K. Li, L.N. Foresee, J.A. Tunge, J. Org. Chem. 70 (2005) 2881-2883; (b) T. Hashimoto, S. Kutubi, T. Izumi, A. Rahman, T. Kitamura, J. Organometal. Chem. 696 (2011) 99-105.

38. For a review, see: (a) M.R. Mohammadizadeh, A. Hasaninejad, M Bahramzadeh, Synth. Commun. 39 (2009) 3232-3242; (b) J. Salazar, S.E Lo' pez, O. Rebollo, J. Fluorine Chem. 124 (2003) 111-113; (c) J. Ohtaka, T. Sakamoto, Y. Kikugawa, Tetrahedron Lett. 50 (2009) 1681-1683; (d) S.E. Lo' pez, Y. Pe' rez, J. Restrepo, J. Salazar, J. Charris, J. Fluorine Chem. 128 (2007) 566-569; (e) F. Herna' ndez-Luis, A. Herna' ndezCampos, R. Castillo, G. Navarrete-Va'zquez, O. Soria-Arteche, M. Herna' ndez-Herna' ndez, L. Ye'pez-Mulia, L. Eur, J. Med. Chem. 45 (2010) 3135-3141.

39. For a review, see: (a) X.-G. Zhang, H.-X. Dai, M. Wasa, J.-Q. Yu, J Am. Chem. Soc. 134 (2012) 11948-11951; (b) M. Eisenberg, D.D Desmarteau, Inorg. Nucl. Chem. Lett. 6 (1970) 29-34; (c) A. Gregoric, M. Zupan, J. Org. Chem. 44 (1979) 4120-4122; (d) T.B. Patrick, K.K. Johli, D.H. White, W.S. Bertrand, R. Mokhtar, M.R. Kilboourn, M.J. Welch, Can. J. Chem. 64 (1986) 138-141; (e) Y. Tanabe, N. Matsuo, N. Ohno, J. Org. Chem. 53 (1988) 4582-4585; (f) N.V. Kondratenko, E.P. Vechirko, L.M. Yagupolskii, Synthesis (1980) 932; (g) F. Cottet, M. Schlosse, Eur. J. Org. Chem. (2002) 327-330.

40. S.S. Sajadikhah, M.T. Maghsoodlou, N. Hazeri, 2013, DOI 10.1007/ s11164-013-1364-0

41. H.-J. Wang, L.-P. Mo, Z.-H. Zhang, ACS Comb. Sci. 13, 181 (2011)

42. S. Rana, M. Brown, A. Bhaumik, C. Mukhopadhyay. Tetrahedron Lett. 54 (2013) 1371-1379.

43. S.S. Sajadikhah, N. Hazeri, M.T. Maghsoodlou, S.M. Habibi-Khorassani, K. Khandan-Barani, J. Chem. Res., (2013) 40.

44. S.S. Sajadikhah, M.T. Maghsoodlou, N. Hazeri, Chin. Chem. Lett., 25 (2014) 58. 